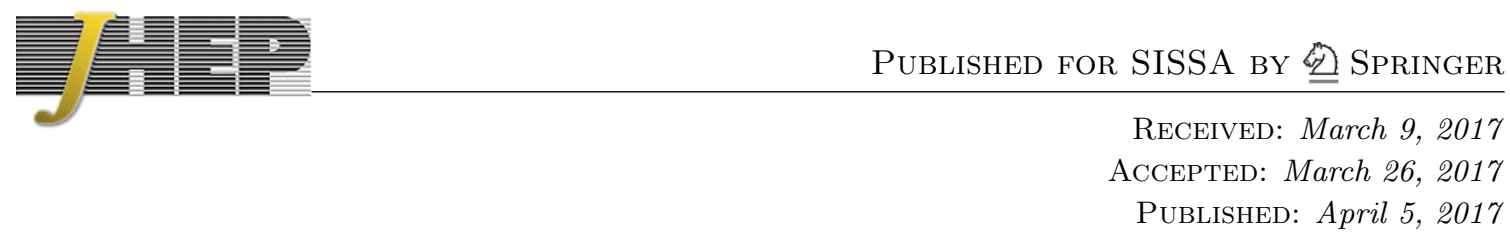

\title{
Constraints on new physics from radiative $B$ decays
}

Ayan Paul ${ }^{a}$ and David M. Straub ${ }^{b}$

${ }^{a}$ INFN, Sezione di Roma, I-00185 Rome, Italy

${ }^{b}$ Excellence Cluster Universe, TUM, Boltzmannstr. 2, 85748 Garching, Germany

E-mail: apaul2@alumni.nd.edu, david.straub@tum.de

ABSTRACT: A new phase for the measurements of radiative decay modes in $b \rightarrow s$ transitions has started with new measurements of exclusive modes by LHCb and with Belle-II showing distinctive promises in both inclusive and exclusive channels. After critically reviewing the hadronic uncertainties in exclusive radiative decays, we analyze the impact of recent measurements of the branching ratio and mass-eigenstate rate asymmetry in $B_{s} \rightarrow \phi \gamma$ and of the angular distribution of $B \rightarrow K^{*} e^{+} e^{-}$at low $q^{2}$ on new physics in the $b \rightarrow s \gamma$ transition.

Keywords: Beyond Standard Model, CP violation, Heavy Quark Physics

ARXIV EPRINT: 1608.02556 


\section{Contents}

1 Introduction $\quad 1$

2 Observables 2

2.1 Effective Hamiltonian 2

2.2 Inclusive radiative decay 3

2.3 Exclusive radiative decays 4

2.3.1 General parametrization of amplitudes 4

2.3.2 Discussion of form factor uncertainties 5

2.3.3 Discussion of hadronic contributions 5

$\begin{array}{lll}2.3 .4 & \text { Exclusive radiative observables } & 7\end{array}$

2.4 Exclusive semi-leptonic angular observables 9

3 Numerical analysis $\quad 9$

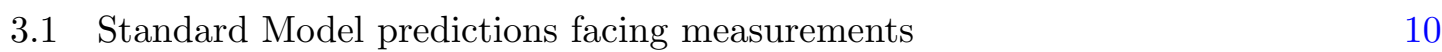

$\begin{array}{ll}3.2 & \text { Constraints on Wilson coefficients } \\ \end{array}$

4 Conclusions and outlook $\quad 14$

$\begin{array}{ll}\text { A Reproducing numerics with flavio } & 16\end{array}$

\section{Introduction}

The radiative flavour-changing neutral current (FCNC) transition $b \rightarrow s \gamma$ is a crucial probe of physics beyond the Standard Model (SM). Its strong sensitivity to new physics (NP) stems from the helicity flip required for the dipole transition, in addition to the CKM and loop suppression. Beyond the SM, new sources of chirality breaking - like the trilinear couplings in the MSSM or masses of heavy vector-like quarks - can strongly modify the $b \rightarrow s \gamma$ transition or can enhance the amplitude with a right-hand polarized photon, $b_{L} \rightarrow s_{R} \gamma_{R}$, that is suppressed by a factor $m_{s} / m_{b}$ at leading order in the SM with respect to $b_{R} \rightarrow s_{L} \gamma_{L}$.

The strongest constraint on the $b \rightarrow s \gamma$ transition comes from the measurement of the branching ratio of the inclusive decay $B \rightarrow X_{s} \gamma$, which is theoretically particularly clean as the decay rate is given simply by the quark-level decay rate in the heavy $b$ quark limit. The branching ratios of the exclusive channels $B^{0,+} \rightarrow K^{*} \gamma$ have been measured even more precisely, but their theoretical estimations are afflicted with considerable hadronic uncertainties. These uncertainties also affect the branching ratio of the exclusive decay $B_{s} \rightarrow \phi \gamma$, that has been measured recently. But branching ratios alone cannot distinguish between the different photon helicities. The most promising observables to make this 
distinction are ones that vanish in the limit of purely left-handed photon polarization. A prominent example is the mixing-induced CP asymmetry in $B^{0} \rightarrow K^{*} \gamma[1,2]$. The angular distribution of the exclusive decay $B \rightarrow K^{*}(\rightarrow K \pi) e^{+} e^{-}$at very low invariant mass of the dielectron pair also offers observables that have this property [3]. Very recently, a previously unmeasured observable of this type has been measured by LHCb [4]: the masseigenstate rate asymmetry $A_{\Delta \Gamma}$ in $B_{s} \rightarrow \phi \gamma$, that quantifies the decay rate difference of the two $B_{s}$ mass eigenstates into the $\phi \gamma$ final state [5]. All these observables have a complementary dependence on the $\mathrm{CP}$ phases and chirality of the new physics contributions and thus lead to powerful constraints on physics beyond the SM, when considered together. Fits of new physics in the $b \rightarrow s \gamma$ transition have been performed in the past in the context of global $b \rightarrow s$ fits (see e.g. [6-9]). Dedicated fits to radiative decays have also been performed $[10,11]$; these have the advantage that they are less dependent on theory uncertainties or potential NP effects in observables that are less sensitive to the radiative dipole transition. The main novelty of our analysis with respect to the latter analyses is that we are including the new measurements of the $B \rightarrow K^{*} e^{+} e^{-}$angular observables at low $q^{2}$ and the $B_{s} \rightarrow \phi \gamma$ mass-eigenstate rate asymmetry for the first time. We also emphasize the importance of the direct CP asymmetry in $B \rightarrow K^{*} \gamma$ as a constraint on new physics. Furthermore, our numerics relies completely on open source codes, which makes it possible for the interested reader to reproduce our numerical predictions and plots and to study the impact of modifying any parametrical assumptions made.

The rest of the paper is organized as follows. In section 2, we define the effective Hamiltonian and discuss the observables in inclusive and exclusive decays that are sensitive to NP. We put a particular emphasis on the theory uncertainties that affect the exclusive decays. In section 3 , we present a numerical analysis of the current constraints on new physics in the $b \rightarrow s \gamma$ transition, taking into account the theoretical uncertainties. Section 4 contains our conclusions.

\section{Observables}

In the following sections we will define our operator basis and then move on to defining the inclusive observables that we use in this work. For discussing the exclusive observables it is important that we include a short discussion on our choice of form factors and how we treat all the hadronic uncertainties that are inherent to these observables. The subtleties of oscillations also appear in some of them and are hence discussed too.

\subsection{Effective Hamiltonian}

The effective Hamiltonian relevant for inclusive and exclusive decays based on the $b \rightarrow s \gamma$ transition in the SM and beyond can be written as ${ }^{1}$

$$
\mathcal{H}_{\mathrm{eff}}=-\frac{4 G_{F}}{\sqrt{2}} \lambda_{t}\left(\sum_{i=1}^{8} C_{i} Q_{i}+\sum_{i=7}^{8} C_{i}^{\prime} Q_{i}^{\prime}\right)
$$

\footnotetext{
${ }^{1}$ For simplicity, we have omitted from $(2.1)$ — but not from our numerics — electroweak penguin operators and doubly Cabibbo-suppressed terms. We also neglect new physics in four-quark operators.
} 
where $\lambda_{i}=V_{i b} V_{i s}^{*}$ and $Q_{1-6}$ are the SM four-quark operators (see e.g. [12] for explicit expressions) and $Q_{7}^{(\prime)}$ and $Q_{8}^{(\prime)}$, the electromagnetic and chromomagnetic dipole operators, are given by

$$
Q_{7}^{(\prime)}=\frac{e}{16 \pi^{2}} m_{b}\left(\bar{s}_{L(R)} \sigma_{\mu \nu} b_{R(L)}\right) F^{\mu \nu}, \quad Q_{8}^{(\prime)}=\frac{g_{s}}{16 \pi^{2}} m_{b}\left(\bar{s}_{L(R)} \sigma_{\mu \nu} T^{a} b_{R(L)}\right) G^{a \mu \nu} .
$$

For the Wilson coefficient $C_{7}$, it is customary to define a regularization scheme independent "effective" coefficient

$$
C_{7}^{\mathrm{eff}}(\mu)=C_{7}(\mu)+\sum_{i=1}^{6} y_{i} C_{i}(\mu)
$$

where $y=\left(0,0,-\frac{1}{3},-\frac{4}{9},-\frac{20}{3},-\frac{80}{9}\right)$ in a scheme with fully anti-commuting $\gamma_{5}$ and in the operator basis used in [13]. Its numerical value at the scale $\mu=4.8 \mathrm{GeV}$ is $C_{7}^{\text {eff }}=-0.2915$ [12], including next-to-next-to-leading order QCD and next-to-leading order electroweak corrections, ${ }^{2}$ with an uncertainty at the per mille level. The chirality-flipped coefficient $C_{7}^{\prime}$ is given by $C_{7}^{\prime}=\frac{m_{s}}{m_{b}} C_{7}$ in the $\mathrm{SM}$.

\subsection{Inclusive radiative decay}

The branching ratio of the inclusive decay $B \rightarrow X_{s} \gamma$ can be predicted to a high accuracy, as the width is given by the width of the quark-level decay $b \rightarrow s \gamma$ up to corrections that vanish in the infinite $b$ quark mass limit. It is to be noted that experiments only resolve photons down to a minimum energy $E_{0}$. At present, the best compromise between experimental and theoretical sensitivity is given by $E_{0}=1.6 \mathrm{GeV}$. To further reduce theoretical uncertainties, the rate can be normalized to the precisely measured semi-leptonic inclusive decay $B \rightarrow$ $X_{c} \ell \nu$, assuming it to be unaffected by NP. The resulting prediction reads

$$
\operatorname{BR}\left(B \rightarrow X_{s} \gamma\right)_{E_{\gamma}>E_{0}}=\operatorname{BR}\left(B \rightarrow X_{c} \ell \nu\right)\left|\frac{\lambda_{t}}{V_{c b}}\right|^{2} \frac{6 \alpha_{\mathrm{em}}}{\pi C}\left[P\left(E_{0}\right)+\delta_{\text {nonp. }}\right]
$$

where $\delta_{\text {nonp. }}$ is a non-perturbative contribution that is estimated at approximately $5 \%$ of the SM branching ratio [15]. At leading order, the function $P\left(E_{0}\right)$ is given by

$$
P\left(E_{0}\right)_{\mathrm{LO}}=\left|C_{7}^{\mathrm{eff}}\right|^{2}+\left|C_{7}^{\prime}\right|^{2} .
$$

Within the SM, corrections of order $\alpha_{s}^{2}$ and $\left(\lambda_{u} / \lambda_{t}\right)^{2}$ have been computed (see [16] for an account on the state of the art).

Apart from the (CP-averaged) branching ratio, also the direct $\mathrm{CP}$ asymmetry in the inclusive decay can be measured and used in principle to constrain CP-violating NP contributions. While the direct $\mathrm{CP}$ asymmetry in $B \rightarrow X_{s} \gamma$ is plagued by poorly known longdistance contributions [17], the direct $\mathrm{CP}$ asymmetry in the untagged decay $B \rightarrow X_{s+d} \gamma$ is free from such contributions and thus theoretically clean. Unless the NP contributions to the Wilson coefficients in the $b \rightarrow d \gamma$ transition are much larger than their counterparts in $b \rightarrow s \gamma$, the NP contribution to the untagged CP asymmetry is dominated by the $b \rightarrow s \gamma$ Wilson coefficients (see e.g. [18]). However, it turns out that even in the presence of NP,

\footnotetext{
${ }^{2}$ See [14] for an account of the contributions to this calculation.
} 
the CP asymmetry is numerically small since the strong phases, generated first at NLO, are small. For instance, we find that even for $\operatorname{Im}\left(C_{7}^{\mathrm{NP}}\right)=0.5$, the untagged CP asymmetry stays below $1 \%$, to be compared to the current world average of $3.2 \pm 3.4 \%$ [19]. We conclude that the inclusive $\mathrm{CP}$ asymmetry currently does not constitute a relevant constraint on new physics.

\subsection{Exclusive radiative decays}

While inclusive decay modes are theoretically cleaner and hence more accurately predictable, exclusive modes are more easily measured in experiments because of their distinctive final states. However, the observables in these exclusive modes are usually shrouded in hadronic uncertainties that are difficult to compute rendering them less conducive for prediction within a theoretical model, be it the SM or a model of new physics. These uncertainties are twofold: on one hand, predicting the observables requires the knowledge of the hadronic form factors of the relevant $B \rightarrow V$ transition. On the other hand, the radiative decays receive contributions where the photon does not participate in the - purely hadronic - hard interaction, a contribution which cannot be expressed in terms of form factors. For predicting these "non-factorizable" contributions, different approaches have been advocated. In the following, we give a general parametrization of these contributions and try to estimate their size and uncertainty, based on existing calculations and estimates.

\subsubsection{General parametrization of amplitudes}

The polarized amplitudes of a general $B_{q} \rightarrow V \gamma$ decay, where $q=u, d$, or $s$, and $V$ is a vector meson, can be written as

$$
\begin{aligned}
& \mathcal{M}\left(\bar{B}_{q} \rightarrow \bar{V} \gamma_{L}\right)=N\left(C_{7}^{\mathrm{eff}} T_{1}(0)+h_{L}\right) S_{L} \\
& \mathcal{M}\left(\bar{B}_{q} \rightarrow \bar{V} \gamma_{R}\right)=N\left(C_{7}^{\prime} T_{1}(0)+h_{R}\right) S_{R} \\
& \mathcal{M}\left(B_{q} \rightarrow V \gamma_{L}\right)=N^{*}\left(C_{7}^{\prime *} T_{1}(0)+h_{R}\right) S_{L} \\
& \mathcal{M}\left(B_{q} \rightarrow V \gamma_{R}\right)=N^{*}\left(C_{7}^{\mathrm{eff} *} T_{1}(0)+h_{L}\right) S_{R}
\end{aligned}
$$

where

$$
\begin{aligned}
N & =-\frac{4 G_{F}}{\sqrt{2}} \lambda_{t} \frac{e}{8 \pi^{2}}, \\
S_{L, R} & =\epsilon^{\mu \nu \rho \sigma} e_{\mu}^{*} \eta_{\nu}^{*} p_{\rho} q_{\sigma} \pm i\left[\left(e^{*} \eta^{*}\right)(p q)-\left(e^{*} p\right)\left(\eta^{*} q\right)\right],
\end{aligned}
$$

$T_{1}(0)$ is the process-dependent $B_{q} \rightarrow V$ tensor form factor at $q^{2}=0$, and we have neglected doubly Cabibbo-suppressed terms of order $\lambda_{u} / \lambda_{t}$. The process-dependent ${ }^{3}$ quantities $h_{L}$ and $h_{R}$ denote contributions of the weak hadronic Hamiltonian, i.e. from the operators $Q_{1-6}$ and $Q_{8}$. In general, they are complex quantities but enter the amplitudes without a conjugate as their phases are CP-even.

\footnotetext{
${ }^{3}$ We omit labels on process-dependent quantities like the form factors or $h_{L, R}$ in the following to avoid clutter.
} 
Equivalently, we can express the hadronic contributions as process-dependent shifts of the Wilson coefficients,

$$
\begin{aligned}
& \mathcal{M}\left(\bar{B}_{q} \rightarrow \bar{V} \gamma_{L(R)}\right)=\left(N \mathcal{C}_{7}^{(\prime)} T_{1}(0)\right) S_{L(R)}, \\
& \mathcal{M}\left(B_{q} \rightarrow V \gamma_{R(L)}\right)=\left(N^{*} \overline{\mathcal{C}}_{7}^{(\prime)} T_{1}(0)\right) S_{R(L)}
\end{aligned}
$$

where we have defined

$$
\begin{aligned}
& \mathcal{C}_{7}=C_{7}^{\mathrm{eff}}+\Delta C_{7}=C_{7}^{\mathrm{eff}}+\frac{h_{L}}{T_{1}(0)} \\
& \overline{\mathcal{C}}_{7}=C_{7}^{\mathrm{eff} *}+\Delta C_{7}=C_{7}^{\mathrm{eff} *}+\frac{h_{L}}{T_{1}(0)}
\end{aligned}
$$

and analogously for the primed coefficient.

\subsubsection{Discussion of form factor uncertainties}

The main source of uncertainty in the branching ratios stems from the form factor $T_{1}(0)$. In our numerical analysis, we use a recent update [20] of a light-cone sum rules calculation [21] of the full QCD form factors yielding

$$
\begin{array}{ll}
T_{1}(0)=0.282 \pm 0.031 & \text { for } B \rightarrow K^{*} \gamma, \\
T_{1}(0)=0.309 \pm 0.027 & \text { for } B_{s} \rightarrow \phi \gamma,
\end{array}
$$

where the form factor is defined at the $\overline{\mathrm{MS}}$ scale $\mu_{b}=4.8 \mathrm{GeV}$. A combined fit of the LCSR results and a recent lattice computation valid at high $q^{2}[22]$ yields

$$
\begin{array}{ll}
T_{1}(0)=0.312 \pm 0.027 & \text { for } B \rightarrow K^{*} \gamma \\
T_{1}(0)=0.299 \pm 0.012 & \text { for } B_{s} \rightarrow \phi \gamma
\end{array}
$$

To be conservative, we will stick to the LCSR result in the following.

\subsubsection{Discussion of hadronic contributions}

Concerning the hadronic effects encoded in $\Delta C_{7}$, we include the following known contributions:

- $O\left(\alpha_{s}\right)$ vertex corrections involving matrix elements of the current-current operators $Q_{1,2}[23]$

- hard spectator scattering involving matrix elements of $Q_{1-6}$ and $Q_{8}$ at leading order in $\Lambda / m_{b}$ from QCD factorization [24];

- weak annihilation at $O\left(\Lambda / m_{b}\right)$ from QCD factorization [25-27].

The numerical central values of all the contributions are shown in table 1 for the three relevant transitions. The vertex correction is by far the largest contribution. At $O\left(\alpha_{s}\right)$, it suffers from a sizable uncertainty due to the dependence on the charm quark mass scheme. This problem is known from the inclusive decay (see e.g. [28, 29]) and could be resolved 
by including $O\left(\alpha_{s}\right)^{2}$ corrections. Importantly, since the vertex correction factorizes into a product of the universal part and a $B \rightarrow V$ form factor, its contribution to the shift $\Delta C_{7}$ is the same for all exclusive decays based on the $b \rightarrow s$ transition. Spectator scattering turns out to be independent of the spectator quark charge at leading order in QCDF, and is, thus, also universal for all three transitions, up to small parametric differences. Our numerical results for the vertex correction and spectator scattering agree well with an alternative derivation using SCET [30]. The weak annihilation contribution is proportional to the spectator quark charge at the order considered, so it differs by a factor -2 between the charged and neutral $B \rightarrow K^{*} \gamma$ transitions. For $B_{s} \rightarrow \phi \gamma$, there is an additional penguin contribution where the $b \rightarrow s$ transition connects the initial $b$ quark with the spectator $s$ quark, but it is suppressed by small Wilson coefficients, so the differences with respect to $B^{0} \rightarrow K^{*} \gamma$ remain small.

The following contributions are instead not included in our central values for $\Delta C_{7}$ :

- QCDF power corrections to spectator scattering involving $Q_{8}$, which are endpoint divergent [25-27];

- Contributions to weak annihilation and spectator scattering involing $Q_{8}$ beyond QCDF that have been computed with light-cone sum rules (LCSR) [31-33];

- Soft gluon corrections to quark loop spectator scattering, in particular the charm loop that comes with a large Wilson coefficient [5, 31, 34].

Of these contributions, the soft gluon correction to the charm loop is expected to be numerically dominant in view of the large Wilson coefficient $C_{2}$. This effect has been estimated for $B \rightarrow K^{*} \gamma$ in [31] using LCSR and finding, in our conventions,

$$
\left.\Delta C_{7}\right|_{\text {soft }}=(-0.52 \pm 0.43) \times 10^{-2},\left.\quad \Delta C_{7}^{\prime}\right|_{\text {soft }}=(0.13 \pm 0.20) \times 10^{-2} .
$$

This calculation was refined and applied to $B_{s} \rightarrow \phi \gamma$ in [5], finding

$$
\left.\Delta C_{7}\right|_{\mathrm{soft}}=(1.11 \pm 0.78) \times 10^{-2} e^{i\left(255 \pm 15^{\circ}\right)},\left.\quad \Delta C_{7}^{\prime}\right|_{\mathrm{soft}}=(0.42 \pm 0.29) \times 10^{-2} e^{i\left(106 \pm 15^{\circ}\right)} .
$$

A different approach, also using LCSR, was taken in [34] finding ${ }^{4}$

$$
\left.\Delta C_{7}\right|_{\mathrm{soft}}=\left(+1.2_{-1.6}^{+0.9}\right) \times 10^{-2},\left.\quad \Delta C_{7}^{\prime}\right|_{\mathrm{soft}} \approx 0 .
$$

Given the marginal agreement between the different estimates, we omit this effect and account for its omission - and all remaining neglected effetcts - by adding an uncertainty on the real and imaginary parts of $\Delta C_{7}$ of $\pm 1.5 \times 10^{-2}$, which includes the central values of all three estimates and is much larger than the difference between the QCDF and LCSR computations of weak annhilation and spectator scattering.

Concerning $\Delta C_{7}^{\prime}$, i.e. hadronic contributions to the "wrong-helicity" amplitude, we do not attempt a prediction but only parametrize our ignorance. While simple power counting

\footnotetext{
${ }^{4}$ The authors of [34] confirmed to us an erroneous sign in their eq. (6.3).
} 


\begin{tabular}{|cccc|}
\hline & $B^{0} \rightarrow K^{*} \gamma$ & $B^{+} \rightarrow K^{*} \gamma$ & $B_{s} \rightarrow \phi \gamma$ \\
\hline Vertex corrections & $-(7.8 \pm 1.0)-(1.1 \pm 0.3) i$ \\
Spectator scattering $Q_{1-6}$ & $-0.7-1.3 i$ & $-0.7-1.3 i$ & $-0.7-1.7 i$ \\
Spectator scattering $Q_{8}$ & -0.3 & -0.3 & -0.4 \\
Weak annihilation & -0.4 & +0.9 & -0.5 \\
\hline
\end{tabular}

Table 1. Contributions to $\Delta C_{7}$ in units of $10^{-2}$ for the three decays. We only show the uncertainties for the (numerically dominant) vertex corrections that are process independent.

leads to a relatively large possible range for this quantity $[35,36]$, based on more refined parametric arguments implying the existence of a helicity suppression $[11,37,38]$ as well as on the above numerical estimates of the soft gluon charm loop contribution to $\Delta C_{7}^{\prime}$ from LCSR, we assume an uncertainty of $\pm 0.4 \times 10^{-2}$ on the real and imaginary parts of $\Delta C_{7}^{\prime}$ and consider this to be a conservative choice.

\subsubsection{Exclusive radiative observables}

With the notation introduced in section 2.3.1, the helicity-summed and CP-averaged branching ratio can be written as

$$
\operatorname{BR}\left(B_{q} \rightarrow V \gamma\right)=\tau_{B_{q}} \frac{G_{F}^{2} \alpha_{\mathrm{em}} m_{B_{q}}^{3} m_{b}^{2}}{32 \pi^{3}}\left(1-\frac{m_{V}^{2}}{m_{B}^{2}}\right)^{3}\left|\lambda^{t}\right|^{2}\left(\left|\mathcal{C}_{7}\right|^{2}+\left|\mathcal{C}_{7}^{\prime}\right|^{2}\right) T_{1}(0),
$$

where the quantities $\alpha_{\mathrm{em}}, m_{b}, \mathcal{C}_{7}^{(\prime)}$, and $T_{1}(0)$ are to be understood as $\overline{\mathrm{MS}}$ quantities at the scale $\mu_{b}$ that we take to be $4.8 \mathrm{GeV}$.

In the decay of the neutral mesons $B_{d}$ and $B_{s}$ to $\mathrm{CP}$ eigenstates, like $B_{s} \rightarrow \phi \gamma$ or $B^{0} \rightarrow K^{*}\left(\rightarrow K_{S} \pi^{0}\right) \gamma$, the time-dependent CP asymmetry leads to additional observables, thanks to meson-antimeson mixing. It reads

$$
\begin{aligned}
A_{\mathrm{CP}}\left(B_{q}(t) \rightarrow V \gamma\right) & =\frac{\Gamma\left(\bar{B}_{q}(t) \rightarrow \bar{V} \gamma\right)-\Gamma\left(B_{q}(t) \rightarrow V \gamma\right)}{\Gamma\left(\bar{B}_{q}(t) \rightarrow \bar{V} \gamma\right)+\Gamma\left(B_{q}(t) \rightarrow V \gamma\right)} \\
& =\frac{S\left(B_{q} \rightarrow V \gamma\right) \sin \left(\Delta M_{q} t\right)+A_{\mathrm{CP}}\left(B_{q} \rightarrow V \gamma\right) \cos \left(\Delta M_{q} t\right)}{\cosh \left(y_{q} t / \tau_{B_{q}}\right)-A_{\Delta \Gamma}\left(B_{q} \rightarrow V \gamma\right) \sinh \left(y_{q} t / \tau_{B_{q}}\right)}
\end{aligned}
$$

containing the direct $\mathrm{CP}$ asymmetry $A_{\mathrm{CP}}$, the mixing-induced CP asymmetry $S$ and the mass-eigenstate rate asymmetry $A_{\Delta \Gamma}$.

In the case of the $B^{0} \rightarrow K^{*} \gamma$, the direct CP asymmetry $A_{\mathrm{CP}}$ also coincides ${ }^{5}$ to excellent approximation with the time-integrated $\mathrm{CP}$ asymmetry, while $A_{\Delta \Gamma}$ can be neglected, since the normalized width difference

$$
y_{q}=\Delta \Gamma_{q} /\left(2 \Gamma_{q}\right)=\tau_{B_{q}} \Delta \Gamma_{q} / 2
$$

is tiny for $B_{d}$ mesons. In terms of the quantities defined above, one can write

$$
A_{\mathrm{CP}}\left(B^{0} \rightarrow K^{*} \gamma\right) \times \mathrm{BR}\left(B^{0} \rightarrow K^{*} \gamma\right)=2 \operatorname{Im} C_{7}^{\text {eff }} \operatorname{Im} \Delta C_{7}+2 \operatorname{Im} C_{7}^{\prime} \operatorname{Im} \Delta C_{7}^{\prime}+\ldots
$$

\footnotetext{
${ }^{5}$ This fact relies on $\Delta M_{d} \ll \Gamma_{d}$ and thus does not hold in the $B_{s}$ system.
} 
where the ellipsis includes in particular the doubly Cabibbo-suppressed SM contribution. Since $\Delta C_{7}^{\prime}$ is expected to be very small, $A_{\mathrm{CP}}$ mostly constrains the imaginary part of the NP contribution to $C_{7}$ [7]. As seen from table 1, spectator scattering induces a non-negligible strong phase to $\Delta C_{7}$, which in combination with the precise measurements of $A_{\mathrm{CP}}$ - that actually do not require a time-dependent analysis - make it a strong constraint as we will discuss in section 3 .

The quantity $A_{\Delta \Gamma}$ is only relevant for the $B_{s}$ decay. Due to the sizable width difference $y_{s} \approx 6 \%$ in the $B_{s}$ system, $A_{\Delta \Gamma}$ can be extracted from the untagged time-dependent decay rate of $B_{s} \rightarrow \phi \gamma$,

$$
\mathrm{BR}\left(B_{s}(t) \rightarrow \phi \gamma\right)=\mathrm{BR}\left(B_{s} \rightarrow \phi \gamma\right) e^{-t / \tau_{B_{s}}}\left[\cosh \left(\frac{y_{s} t}{\tau_{B_{s}}}\right)-A_{\Delta \Gamma}\left(B_{s} \rightarrow \phi \gamma\right) \sinh \left(\frac{y_{s} t}{\tau_{B_{s}}}\right)\right] .
$$

This also implies that the time-integrated branching ratio of the $B_{s} \rightarrow \phi \gamma$ decay measured experimentally does not coincide with the theoretical branching ratio in the absence of $B_{s}-\bar{B}_{s}$ mixing given in (2.20). The two quantities are related as

$$
\overline{\mathrm{BR}}\left(B_{s} \rightarrow \phi \gamma\right)=\left[\frac{1-A_{\Delta \Gamma}\left(B_{s} \rightarrow \phi \gamma\right) y_{s}}{1-y_{s}^{2}}\right] \operatorname{BR}\left(B_{s} \rightarrow \phi \gamma\right)
$$

Particularly simple expressions for $S$ and $A_{\Delta \Gamma}$ can be obtained in the approximation $\left|\mathcal{C}_{7}^{(\prime)}\right| \approx\left|\overline{\mathcal{C}}_{7}^{(\prime)}\right|$ (the violation of this relation generates $A_{\mathrm{CP}}$ ). Then one can write $\mathcal{C}_{7}=$ $\left|\mathcal{C}_{7}\right| e^{i \phi_{7}} e^{i \delta_{7}},\left|\overline{\mathcal{C}}_{7}\right|=\left|\mathcal{C}_{7}\right| e^{-i \phi_{7}} e^{i \delta_{7}}$, and analogously for the primed coefficients, leading to

$$
\begin{aligned}
S\left(B_{s} \rightarrow \phi \gamma\right) & =\sin (2 \chi) \sin \left(\phi_{7}+\phi_{7}^{\prime}-\phi_{s}^{\Delta}\right) \cos \left(\delta_{7}-\delta_{7}^{\prime}\right), \\
A_{\Delta \Gamma}\left(B_{s} \rightarrow \phi \gamma\right) & =\sin (2 \chi) \cos \left(\phi_{7}+\phi_{7}^{\prime}-\phi_{s}^{\Delta}\right) \cos \left(\delta_{7}-\delta_{7}^{\prime}\right),
\end{aligned}
$$

where we have introduced

$$
\tan \chi \equiv\left|\frac{\mathcal{C}_{7}^{\prime}}{\mathcal{C}_{7}}\right|
$$

and $\phi_{s}^{\Delta}$ is a NP contribution to the $B_{s}$ mixing phase. Analogously, the mixing-induced CP asymmetry in $B^{0} \rightarrow K^{*}\left(\rightarrow K_{S} \pi^{0}\right) \gamma$ is given by

$$
S\left(B^{0} \rightarrow K^{*} \gamma\right)=\sin (2 \chi) \sin \left(\phi_{7}+\phi_{7}^{\prime}-2 \beta-\phi_{d}^{\Delta}-2\left|\beta_{s}\right|\right) \cos \left(\delta_{7}-\delta_{7}^{\prime}\right),
$$

where $\beta=\arg \left(V_{t b}^{*} V_{t d}\right), \beta_{s}=\arg \left(V_{t b}^{*} V_{t s}\right)$. While we do not use these approximate expressions in our numerics, they demonstrate clearly that

- both $S\left(B^{0} \rightarrow K^{*} \gamma\right)$ and $A_{\Delta \Gamma}\left(B_{s} \rightarrow \phi \gamma\right)$ measure the ratio of the amplitudes with right- and left-handed photons,

- in the case of NP contributions leading to a sizable value for these observables, the dependence on the strong phases is small, as they only enter in the cosine terms.

The latter is in constrast to the direct $\mathrm{CP}$ asymmetry in $B \rightarrow K^{*} \gamma$ which is instead strongly dependent on the strong phases as seen in (2.24). 


\subsection{Exclusive semi-leptonic angular observables}

While strictly speaking $B^{0} \rightarrow K^{* 0}(\rightarrow K \pi) e^{+} e^{-}$is not a radiative decay, the angular analysis of this rare semi-leptonic decay at a very low dilepton invariant mass squared can give complementary constraints on the Wilson coefficients $C_{7}$ and $C_{7}^{\prime}$. This is true in particular for the observables [3, 39-41]

$$
P_{1}=A_{T}^{(2)}=\frac{S_{3}}{2 S_{2}^{s}}, A_{T}^{(\mathrm{Im})}=\frac{A_{9}}{2 S_{2}^{s}},
$$

where we use the conventions used by the LHCb collaboration (see e.g. [12] for a dictionary between different conventions). $P_{1}$ is a CP-averaged angular observable and $A_{T}^{(\mathrm{Im})}$ a T-odd $\mathrm{CP}$ asymmetry. Both observables are quasi null tests of the SM. Importantly, both only depend on the transverse $B \rightarrow K^{*} e^{+} e^{-}$helicity amplitudes. These helicity amplitudes also receive contributions from the weak hadronic Hamiltonian, but for $q^{2} \rightarrow 0$ they coincide with the contributions to the $B^{0} \rightarrow K^{*} \gamma$ amplitudes. Using again the approximation $\left|\mathcal{C}_{7}^{(\prime)}\right| \approx\left|\overline{\mathcal{C}}_{7}^{(\prime)}\right|$, one can thus write (cf. [11])

$$
\begin{aligned}
\lim _{q^{2} \rightarrow 0} P_{1} & =\sin (2 \chi) \cos \left(\phi_{7}-\phi_{7}^{\prime}\right) \cos \left(\delta_{7}-\delta_{7}^{\prime}\right), \\
\lim _{q^{2} \rightarrow 0} A_{T}^{(\mathrm{Im})} & =\sin (2 \chi) \sin \left(\phi_{7}-\phi_{7}^{\prime}\right) \cos \left(\delta_{7}-\delta_{7}^{\prime}\right),
\end{aligned}
$$

where $\chi$ has been defined in (2.29).

In practice, the observables are measured in finite $q^{2}$ bins, as dictated by the experimental resolution and statistical precision. While (2.32) and (2.33) no longer hold exactly in this case, the impact of Wilson coefficients other than the ones contributing to radiative decays is marginal even in the presence of NP, taking into account other constraints.

Interestingly, the expressions (2.32) and (2.33) are very similar to the expressions (2.28) and (2.27) for $A_{\Delta \Gamma}$ and $S\left(B_{s} \rightarrow \phi \gamma\right)$, but there is an important difference: while the latter depend on the sum of the weak phases of $\mathcal{C}_{7}$ and $\mathcal{C}_{7}^{\prime}$, the former depend on their difference. In scenarios with complex contributions to both Wilson coefficients, they are thus complementary. Moreover, the semi-leptonic angular observables are not affected by NP in meson-antimeson mixing.

Before proceding to the numerical analysis, a comment is in order about the processes not discussed so far. While baryonic exclusive radiative decays such as $\Lambda_{b} \rightarrow \Lambda \gamma$ are promising but still awaiting to be measured, LHCb has recently measured [42] a triple product asymmetry in $B \rightarrow K_{1}(\rightarrow K \pi \pi) \gamma$ that is sensitive to the photon polarization [4345]. However at present this observable does not constitute a relevant constraint on NP as it is plagued by large hadronic uncertainties. In our notation, this asymmetry is proportional to $\cos (2 \chi)$, so it is less sensitive to small $\chi$ than the observables discussed above.

\section{$3 \quad$ Numerical analysis}

In our numerical analysis we will primarily use flavio [50] and cross-check all the results with HEPfit [51]. While all the analysis in this work will be made available as a standard 


\begin{tabular}{|c|c|c|c|c|}
\hline Observable & SM prediction & & Measurement & \\
\hline $10^{4} \times \mathrm{BR}\left(B \rightarrow X_{s} \gamma\right)_{E_{\gamma}>1.6 \mathrm{GeV}}$ & $3.36 \pm 0.23$ & {$[16]$} & $3.27 \pm 0.14$ & {$[46]$} \\
\hline $10^{5} \times \mathrm{BR}\left(B^{+} \rightarrow K^{*} \gamma\right)$ & $3.43 \pm 0.84$ & & $4.21 \pm 0.18$ & {$[19]$} \\
\hline $10^{5} \times \mathrm{BR}\left(B^{0} \rightarrow K^{*} \gamma\right)$ & $3.48 \pm 0.81$ & & $4.33 \pm 0.15$ & {$[19]$} \\
\hline $10^{5} \times \overline{\mathrm{BR}}\left(B_{s} \rightarrow \phi \gamma\right)$ & $4.31 \pm 0.86$ & & $3.5 \pm 0.4$ & {$[47,48]$} \\
\hline$S\left(B^{0} \rightarrow K^{*} \gamma\right)$ & $-0.023 \pm 0.015$ & & $-0.16 \pm 0.22$ & {$[19]$} \\
\hline$A_{\mathrm{CP}}\left(B^{0} \rightarrow K^{*} \gamma\right)$ & $0.003 \pm 0.001$ & & $-0.002 \pm 0.015$ & {$[19]$} \\
\hline$A_{\Delta \Gamma}\left(B_{s} \rightarrow \phi \gamma\right)$ & $0.031 \pm 0.021$ & & $-1.0 \pm 0.5$ & {$[4]$} \\
\hline$\left\langle P_{1}\right\rangle\left(B^{0} \rightarrow K^{*} e^{+} e^{-}\right)_{[0.002,1.12]}$ & $0.04 \pm 0.02$ & & $-0.23 \pm 0.24$ & {$[49]$} \\
\hline$\left\langle A_{T}^{\operatorname{Im}}\right\rangle\left(B^{0} \rightarrow K^{*} e^{+} e^{-}\right)_{[0.002,1.12]}$ & $0.0003 \pm 0.0002$ & & $0.14 \pm 0.23$ & {$[49]$} \\
\hline
\end{tabular}

Table 2. SM predictions vs. experimental world averages of observables sensitive to the $b \rightarrow$ $s \gamma$ transition.

part of a flavio release, the necessary modifications for HEPfit, tuned to match the numerics generated by flavio, can be made available on request. The details of the flavio implementation can be found in appendix A. In the following sections we will look at the SM predictions for the observables that we have discussed previously. We will also see how these observables can constrain contributions to $C_{7}$ and $C_{7}^{\prime}$ generated by new physics. As a separate exercise we try to fit for the values of the form factors assuming the experimental numbers to be SM signals.

\subsection{Standard Model predictions facing measurements}

The $\mathrm{CP}$ and isospin averaged branching ratio of the inclusive $B \rightarrow X_{s} \gamma$ decay has been measured at CLEO [52], Belle [53, 54] and BaBar [55-58]. The HFAG world average for a minimum photon energy of $1.6 \mathrm{GeV}$ is shown in table 2 and is in excellent agreement with the SM prediction shown in the same table.

Measurements of the exclusive $B \rightarrow K^{*} \gamma$ branching ratio have been reported by CLEOII [59], Belle [60] and BaBar [61]. The current HFAG world averages for the charged and neutral modes are shown in table 2 along with our SM predictions. ${ }^{6}$

The first measurement of the (time-integrated) branching ratio of $B_{s} \rightarrow \phi \gamma$ has been performed by Belle [63]. LHCb has presented a measurement of the ratio of branching ratios of $B_{s} \rightarrow \phi \gamma$ and $B^{0} \rightarrow K^{*} \gamma$ [47], which can be converted to a measurement of $\mathrm{BR}\left(B_{s} \rightarrow \phi \gamma\right)$ by using the world average of $\mathrm{BR}\left(B^{0} \rightarrow K^{*} \gamma\right)$ from other experiments. Again, table 2 compares our SM prediction with the HFAG world average obtained in this way.

\footnotetext{
${ }^{6}$ By including the branching ratios of both the charged and neutral mode, we implicitly include the isospin asymmetry as a constraint, which is generated by the interference of weak annihilation and $C_{7}$. Note however that the current HFAG averages do not take into account the different treatment of the $B^{+} / B^{0}$ production asymmetry at $B$ factories, which leads to a bias on the asymmetry [62].
} 
Comparing the SM predictions with the measurements of the three exclusive branching ratios, one notices that, although they agree at the level of $1 \sigma$, the SM predictions tend to be on the low side for $B \rightarrow K^{*} \gamma$ and on the high side for $B_{s} \rightarrow \phi \gamma$. Such a pattern - if it were significant - could not be explained by NP, which would affect both decays in the same way. Likewise, it is unlikely to be explained by a hadronic effect encoded in $\Delta C_{7}^{(\prime)}$ since it would either affect all three branching ratios in a similar way (for spectator-independent effects) or one would expect a larger difference between the $B^{+}$and $B^{0}$ decay than between the $B^{0}$ and $B_{s}$ decay (for effects depending on the spectator charge). Consequently, if this pattern persists with more precise measurements, it would point towards a higher value for the $B \rightarrow K^{*}$ form factor $T_{1}(0)$ and a lower value for the corresponding $B_{s} \rightarrow \phi$ form factor than in eqs. (2.15a) and (2.15b), respectively.

To quantify this statement, we have performed two Bayesian fits of the relevant hadronic quantities to the measured exclusive branching ratios $\mathrm{BR}\left(B^{0} \rightarrow K^{*} \gamma\right), \operatorname{BR}\left(B^{+} \rightarrow\right.$ $\left.K^{*} \gamma\right)$, and $\operatorname{BR}\left(B_{s} \rightarrow \phi \gamma\right)$ using a Markov Chain Monte Carlo (with flavio and emcee [64]), assuming the validity of the SM. For the first fit, we assumed flat priors for the form factors $T_{1}(0)$, for the second fit we used the LCSR priors, (2.15). The posterior distribution for the form factors for both fits are shown in figure 1, compared to the LCSR prior. For the fit with flat priors, we find the following mean and variance of the form factors,

$$
\begin{array}{ll}
T_{1}(0)=0.316_{-0.015}^{+0.016} & \text { for } B \rightarrow K^{*} \gamma, \\
T_{1}(0)=0.280_{-0.022}^{+0.020} & \text { for } B_{s} \rightarrow \phi \gamma .
\end{array}
$$

These can be seen as fit predictions given the data, if the SM holds.

The mixing-induced CP asymmetry $S_{K^{*} \gamma}$ has been measured ${ }^{7}$ by both Belle and BaBar $[65,66]$, the HFAG average is shown in table 2. While these measurements are still far from the small SM prediction, Belle-II is expected to measure $S_{K^{*} \gamma} \sim 3 \%$ with 50 $a b^{-1}$ of data [67].

The direct CP asymmetry $A_{\mathrm{CP}}\left(B^{0} \rightarrow K^{*} \gamma\right)$ has been measured on the one hand in the time-dependent CP asymmetry in $B^{0} \rightarrow K^{*}\left(\rightarrow K_{S} \pi^{0}\right) \gamma$ by BaBar and Belle. On the other hand, as discussed in section 2.3.4, it coincides with the time-integrated $\mathrm{CP}$ asymmetry, which has been measured much more precisely. The HFAG world average shown in table 2 includes measurements from BaBar [61] and LHCb [47].

Angular observables of the decay $B^{0} \rightarrow K^{*} e^{+} e^{-}$at low $q^{2}$ have been measured only by LHCb so far [49]. The same holds for the mass-eigenstate rate asymmetry $A_{\Delta \Gamma}$ in $B_{s} \rightarrow \phi \gamma$, that has been measured very recently for the first time [4], with a large negative central value but still large uncertainty. While this measurement is two standard deviations away from the SM expectation, we stress that values below -1 are actually unphysical as seen from (2.28).

For all the observables that vanish in the limit of purely left-handed photon polarization, i.e. $S\left(B^{0} \rightarrow K^{*} \gamma\right), A_{\Delta \Gamma}\left(B_{s} \rightarrow \phi \gamma\right)$, and the binned angular observables $\left\langle P_{1}\right\rangle$ and $\left\langle A_{T}^{\mathrm{Im}}\right\rangle$ in $B^{0} \rightarrow K^{*} e^{+} e^{-}$, the measurements are still far away from the small SM predictions. From eqs. (2.30), (2.28), (2.32), and (2.33), it can be seen that the SM predictions

\footnotetext{
${ }^{7}$ Belle and BaBar have also measured $S_{K_{S} \pi^{0} \gamma}$ including the resonant regions of $K^{*} \gamma$. The HFAG average for this quantity is $S_{K_{S} \pi^{0} \gamma}=-0.15 \pm 0.20$. We do not use it in our numerics.
} 


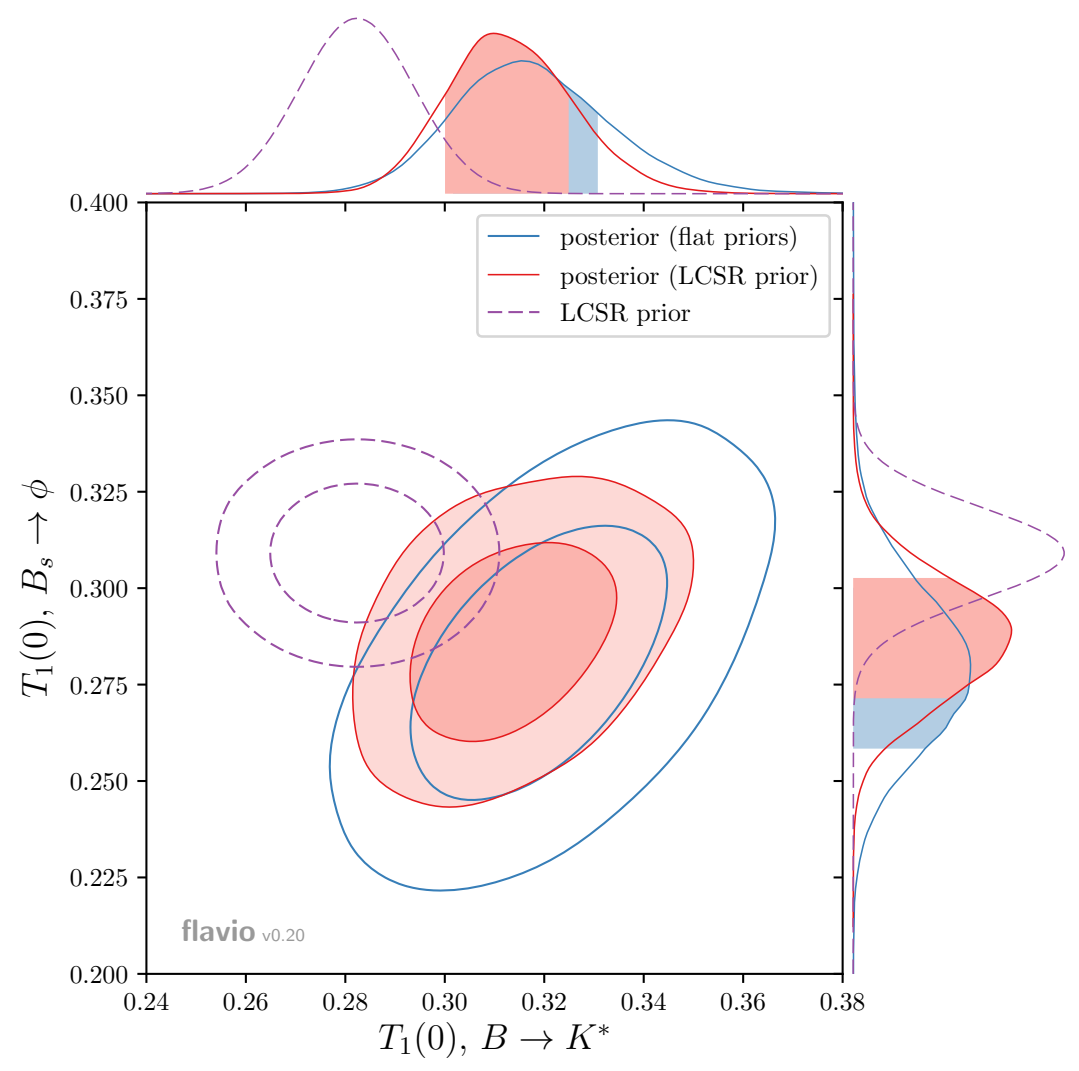

Figure 1. Posterior probability distribution of the tensor form factors $T_{1}(0)$ in $B \rightarrow K^{*}$ and $B_{s} \rightarrow \phi$ transitions from two Bayesian fits to the three exclusive radiative branching ratios, assuming flat priors (blue, empty contours) or the LCSR priors, (2.15) (red, filled contours). The dashed contours show the LCSR priors, (2.15), for comparison. Contours correspond to 68.3 and $95.5 \%$ posterior probability. The curves along the top and right axes show the one-dimensional marginal (posterior or prior) distributions for the individual form factors; the shaded bands correspond to $68.3 \%$ marginal probability.

are proportional to $\mathcal{C}_{7}^{\prime}=\left(m_{s} / m_{b}\right) C_{7}+\Delta C_{7}$ and thus depend crucially on the assumption made on the hadronic contribution $\Delta C_{7}$, that we have discussed in section 2.3.3.

\subsection{Constraints on Wilson coefficients}

To constrain the parameter space of NP models, it is crucial to know the allowed values of the NP contributions to the Wilson coefficients $C_{7}$ and $C_{7}^{\prime}$. In this section, we discuss the constraints separately for the real and imaginary parts of $C_{7}$ as well as for $C_{7}^{\prime}$.

Real part of $\boldsymbol{C}_{\mathbf{7}}$. In scenarios where $C_{7}^{\prime}$ does not receive NP contributions and the contribution to $C_{7}$ is aligned in phase with the $\mathrm{SM}$, the only observables sensitive to NP among the ones we have discussed are the branching ratios. Figure 2 shows the constraints on NP contributions to the real part of $C_{7}$ from the individual exclusive and the inclusive branching ratio, as well as the global constraint. The latter is of course dominated by the inclusive branching ratio, which has the smallest theory uncertainty. We find the following 


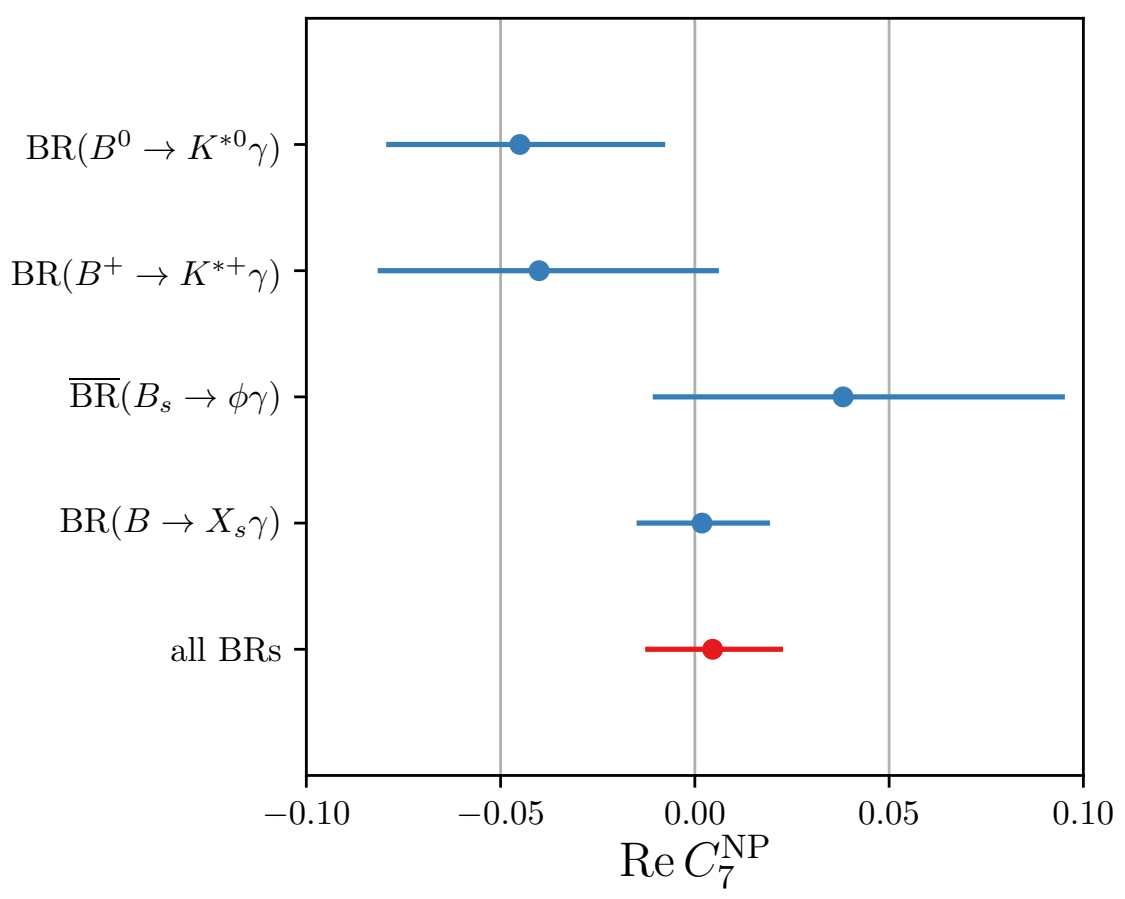

Figure 2. Constraint on NP contributions to the real part of the Wilson coefficient $C_{7}$ from exclusive and inclusive branching ratios as well as combined constraint from these branching ratios.

best-fit regions for the NP contribution, ${ }^{8}$

$$
\operatorname{Re} C_{7}^{\mathrm{NP}}\left(\mu_{b}\right) \in \begin{cases}{[-0.018,0.012]} & @ 1 \sigma, \\ {[-0.032,0.027]} & @ 2 \sigma,\end{cases}
$$

where $\mu_{b}=4.8 \mathrm{GeV}$.

Imaginary part of $\boldsymbol{C}_{\mathbf{7}}$. As discussed in section 2.3.4, the only stringent constraint on the imaginary part of $C_{7}^{\mathrm{NP}}$ is expected to come from $A_{\mathrm{CP}}\left(B \rightarrow K^{*} \gamma\right)$. Using the experimental measurement in table 2, we find

$$
\operatorname{Im} C_{7}^{\mathrm{NP}}\left(\mu_{b}\right) \in[-0.064,0.094] \times\left[\frac{-0.027}{\operatorname{Im} \Delta C_{7}}\right] @ 95 \% \text { C.L. }
$$

Using our numerics and theory error estimates detailed in section 2.3.3, we find

$$
\operatorname{Im} \Delta C_{7}\left(\mu_{b}\right)=-0.027 \pm 0.016 \quad \text { for } B^{0} \rightarrow K^{*} \gamma,
$$

where the central value is dominated by vertex corrections and spectator scattering (cf. table 1) and the uncertainty by our estimate of neglected contributions, including the soft gluon correction to the charm loop. From (3.5) it is clear that an accidental cancellation in the imaginary part of $\Delta C_{7}$, that would make $A_{\mathrm{CP}}$ tiny even in the presence of NP in

\footnotetext{
${ }^{8}$ With the one-dimensional 1 and $2 \sigma$ regions, we refer to regions where the logarithm of the likelihood is within 1 and 4 of the best-fit value, respectively.
} 
$\operatorname{Im} C_{7}$, is not entirely excluded. We note that the estimate of the soft gluon contribution in (2.18), that we omitted, would make the constraint even stronger. In any case, a better understanding of the hadronic contributions is crucial to better constrain this Wilson coefficient.

Constraints on $C_{7}^{\prime}$. The virtues of the exclusive observables come to play in models predicting a NP contribution to the "wrong-chirality" Wilson coefficient $C_{7}^{\prime}$. In figure 3 , we show the constraints in the plane of NP contributions to $\operatorname{Re} C_{7}$ vs. $\operatorname{Re} C_{7}^{\prime}$ and $\operatorname{Re} C_{7}^{\prime}$ vs. $\operatorname{Im} C_{7}^{\prime}$. The contours correspond to constant values of $\Delta \chi^{2}$ with respect to a best fit point, obtained by combining (correlated) experimental and theoretical uncertainties. ${ }^{9}$ In each of the plots, we have assumed NP to only affect the two quantities plotted (e.g., in the first plot, both coefficients are assumed to be real). In addition to the global 1 and $2 \sigma$ constraints, we also show the $1 \sigma$ constraints from individual exclusive observables as well as from the combination of all branching ratios. These plots highlight the complementarity of the exclusive observables: while the imaginary part of $C_{7}^{\prime}$ is constrained by $A_{T}^{\mathrm{Im}}$, the real part is constrained by $A_{\Delta \Gamma}$ and $P_{1}$, while $S_{K^{*} \gamma}$ leads to a constraint in the complex $C_{7}^{\prime}$ plane that is "rotated" by the $B^{0}$ mixing phase $2 \beta$. The new measurement of $A_{\Delta \Gamma}$ shows a preference for non-zero $\operatorname{Re} C_{7}^{\prime}$, but given its large uncertainties, it is not in disagreement with the measurement of $P_{1}$.

Since the experimental central value of $A_{\Delta \Gamma}$ is at the border of the physical domain, we provide best fit values and correlated errors on the real and imaginary parts of $C_{7}^{\prime}$ in a fit without $A_{\Delta \Gamma}$ and in a fit including it, obtained by approximating the likelihood in the vicinity of the best fit point as a multivariate Gaussian. We find

$$
\begin{array}{ll}
\left(\begin{array}{c}
\operatorname{Re} C_{7}^{\prime N P}\left(\mu_{b}\right) \\
\operatorname{Im} C_{7}^{\prime}\left(\mu_{b}\right)
\end{array}\right)=\left(\begin{array}{l}
0.018 \pm 0.037 \\
0.001 \pm 0.037
\end{array}\right), & \left.\rho=0.34 \quad \text { (without } A_{\Delta \Gamma}\right), \\
\left(\begin{array}{c}
\operatorname{Re} C_{7}^{\prime N P}\left(\mu_{b}\right) \\
\operatorname{Im} C_{7}^{\prime}\left(\mu_{b}\right)
\end{array}\right)=\left(\begin{array}{l}
0.038 \pm 0.035 \\
0.006 \pm 0.036
\end{array}\right), & \rho=0.29 \quad\left(\text { with } A_{\Delta \Gamma}\right),
\end{array}
$$

where $\rho$ are the correlation coefficients.

\section{Conclusions and outlook}

The $b \rightarrow s \gamma$ transition belongs to the most important probes of NP in the flavour sector. While the most stringent constraint on new contributions with left-handed photon helicity comes from the branching ratio of the inclusive decay $B \rightarrow X_{s} \gamma$, the exclusive radiative decays $B_{q} \rightarrow V \gamma$ and the semileptonic decays $B_{q} \rightarrow V e^{+} e^{-}$at low $q^{2}$ are complementary probes that are sensitive to the photon helicity. In this paper, after having critically reviewed all the hadronic uncertainties in exclusive radiative and semi-leptonic decays, we have updated the numerical analysis of new physics in the Wilson coefficients $C_{7}$ and $C_{7}^{\prime}$ of the electromagnetic dipole operators, taking into account the recent measurements of the $B \rightarrow K^{*} e^{+} e^{-}$angular distribution and the untagged time-dependent $B_{s} \rightarrow \phi \gamma$ decay rate by $\mathrm{LHCb}$ and emphasizing the role played by the direct CP asymmetry in $B \rightarrow K^{*} \gamma$.

\footnotetext{
${ }^{9}$ See $[7]$ and the documentation of the FastFit class in flavio for details on the procedure.
} 

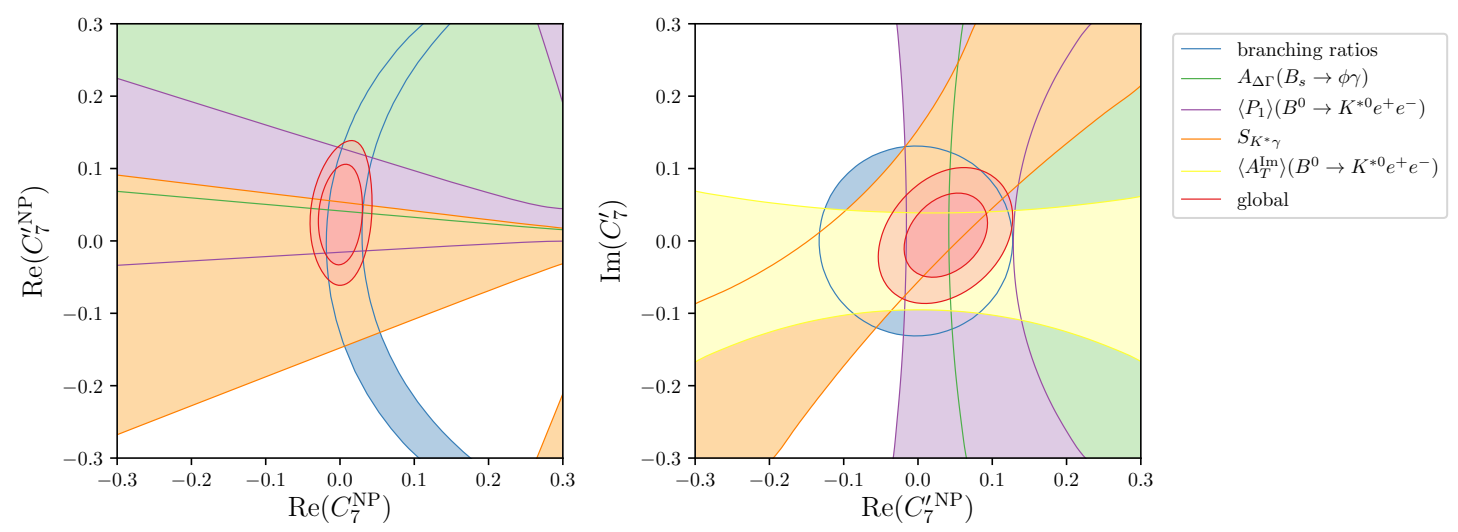

Figure 3. Constraints on NP contributions to the Wilson coefficients $C_{7}$ and $C_{7}^{\prime}$. For the global constraints, 1 and $2 \sigma$ contours are shown, while the individual constraints are shown at $1 \sigma$ level.

Our main findings can be summarized as follows.

- The inclusive and exclusive branching ratios strongly constrain NP contributions to the real part of $C_{7}$, cf. (3.3).

- Assuming the SM to hold, the exclusive branching ratios can also be used to extract the form factors, cf. (3.2).

- The observable most sensitive to the imaginary part of $C_{7}$ is the direct $\mathrm{CP}$ asymmetry in $B \rightarrow K^{*} \gamma$, cf. (3.4). However, the contribution is proportional to the sine of a strong phase that is rather uncertain, adopting our conservative error estimates. Improved determinations of this phase would be useful to better constrain this Wilson coefficient.

- The Wilson coefficient $C_{7}^{\prime}$ is constrained by a number of theoretically clean ${ }^{10}$ observables with complementary dependence on the Wilson coefficients: the mixing-induced $\mathrm{CP}$ asymmetry in $B \rightarrow K^{*} \gamma$, the angular observables $P_{1}$ and $A_{T}^{\operatorname{Im}}$ in $B \rightarrow K^{*} e^{+} e^{-}$ at low $q^{2}$, and the mass-eigenstate rate asymmetry $A_{\Delta \Gamma}$ in $B_{s} \rightarrow \phi \gamma$ measured recently for the first time. The new measurement of $A_{\Delta \Gamma}$ shows a slight preference for non-standard $C_{7}^{\prime}$, but the global fit does not show a significant tension.

While we have only presented one- and two-dimensional constraints on Wilson coefficients, a global Bayesian fit simultaneously fitting all Wilson coefficients as well as the hadronic contributions would be interesting to quantify the agreement of different hypothesis on NP or hadronic contributions given the data. We leave this exercise to a future update of this work.

In our numerical analysis, we have purely relied on open source codes, in particular flavio ${ }^{11}$ and HEPfit. ${ }^{12}$ appendix A contains some details on how to modify flavio to

\footnotetext{
${ }^{10}$ Given present experimental uncertainties.

${ }^{11}$ https://flav-io.github.io.

${ }^{12}$ http://hepfit.roma1.infn.it.
} 
study the impact of different parameter choices. These public codes can also play an instrumental role in improving the constraints on new physics in $C_{7}^{(\prime)}$ by future measurements, e.g. by LHCb or Belle-II. In addition to improved measurements of the $B \rightarrow K^{*} \gamma$ and $B_{s} \rightarrow \phi \gamma$ branching ratios, this includes in particular

- measurements of the radiative baryonic decays $\Lambda_{b} \rightarrow \Lambda^{(*)} \gamma[68,69]$,

- a more precise measurement of the time-dependent CP asymmetry in $B^{0} \rightarrow K^{*} \gamma$ to zero in on $S_{K^{*} \gamma}$,

- Improved measurements of the $B \rightarrow K^{*} e^{+} e^{-}$angular analysis at very low $q^{2}$ and the analogous measurement in $B_{s} \rightarrow \phi e^{+} e^{-}$.

On the theory side, the main limiting factor in exclusive decays is the form factor uncertainty, impeding the exploitation of the precise branching ratio measurements. A moderate improvement might be possible in the future from extrapolations of improved lattice calculations of the form factors at high $q^{2}$, in particular in the $B_{s} \rightarrow \phi$ case, while the $B \rightarrow K^{*}$ form factors are more challenging due to the large $K^{*}$ width [70]. Concerning angular observables, mixing-induced $\mathrm{CP}$ asymmetries and $A_{\Delta \Gamma}$, these observables are instead virtually unaffected by the form factor uncertainty. Their uncertainty is dominated by poorly known contributions to the hadronic quantities $\Delta C_{7}^{(\prime)}$ that would profit from more precise estimates in the future. Nevertheless, given their smallness within the SM, these uncertainties will be subdominant compared to the experimental uncertainties for the next few years, unless a sizable deviation from the SM expectation is observed.

\section{Acknowledgments}

D. S. thanks Wolfang Altmannshofer, Christoph Bobeth, Martino Borsato, Martin Jung, Alexander Khodjamirian, Mikołaj Misiak, Marie-Hélène Schune and Roman Zwicky for useful discussions and Thomas Blake for an important bug report on flavio. The work of D. S. is supported by the DFG cluster of excellence "Origin and Structure of the Universe". A. P. would like to thank Emi Kou for discussions related to this work. A. P. is supported by the European Research Council under the European Union's Seventh Framework Programme (FP/2007-2013)/ERC Grant Agreement n. 279972 'NPFlavour'.

\section{A Reproducing numerics with flavio}

The Standard Model predictions and plots in this paper have been obtained with the open source Python package flavio, version 0.20. For usage documentation and details on this code, see its web site. ${ }^{13}$ The central values and uncertainties of all parameters discussed in this paper correspond to the default values of this version, with the exception of the $B \rightarrow V$ form factors, that we take from a LCSR calculation, while flavio by default uses a combined fit to lattice and LCSR results. The LCSR form factors can be loaded as default in any script or session, after invoking import flavio, with the commands

from flavio.physics.bdecays.formfactors.b_v import bsz_parameters

bsz_parameters.bsz_load_v2_lcsr(flavio.default_parameters)

\footnotetext{
${ }^{13}$ https://flav-io.github.io.
} 
The SM central values and uncertainties of the radiative decay observables can be computed with the commands

flavio.sm_prediction(<obs>)

flavio.sm_uncertainty (<obs>)

where <obs> has to be replaced by

- 'BR (B->Xsgamma)' for $\mathrm{BR}\left(B \rightarrow X_{s} \gamma\right)_{E_{\gamma}>1.6 \mathrm{GeV}}$,

- 'BR(B0->K*gamma)' for $\operatorname{BR}\left(B^{0} \rightarrow K^{* 0} \gamma\right)$,

- 'BR (B+->K*gamma)' for $\mathrm{BR}\left(B^{+} \rightarrow K^{*+} \gamma\right)$,

- 'BR(Bs->phigamma)' for $\overline{\mathrm{BR}}\left(B_{s} \rightarrow \phi \gamma\right)$,

- 'S_K*gamma' for $S_{K^{*} \gamma}$,

- 'ADeltaGamma (Bs->phigamma)' for $A_{\Delta \Gamma}\left(B_{s} \rightarrow \phi \gamma\right)$.

For the $B \rightarrow K^{*} e^{+} e^{-}$observables, the analogous commands read

flavio.sm_prediction(<obs>, q2min=0.002, q2max=1.12)

flavio.sm_uncertainty(<obs>, q2min=0.002, q2max $=1.12$ )

where $\langle o b s\rangle$ is

- ' $\left\langle\mathrm{P} 1>(\mathrm{B} 0->\mathrm{K} * \mathrm{ee})\right.$ ' for $\left\langle P_{1}\right\rangle$,

- ' $\left\langle\mathrm{ATIm}>(\mathrm{B} 0->\mathrm{K} * \mathrm{ee})\right.$ ' for $\left\langle A_{T}^{\operatorname{Im}}\right\rangle$.

The easiest way to study the impact of different parameter or theory uncertainty choices is to modify the default parameter values. For instance, to set the $B \rightarrow K^{*}$ form factor $T_{1}(0)$ to $0.3 \pm 0.1$, use

flavio.default_parameters.set_constraint('B->K* BSZ a0_T1', '0.3 +- 0.1')

The other most relevant parameters for exclusive radiative decays are

- 'Bs->phi BSZ a0_T1' $-B_{s} \rightarrow \phi$ form factor $T_{1}(0)$

- 'B0->K*0 deltaC7p a_+ $\operatorname{Re}{ }^{\prime}-\operatorname{Re}\left(\Delta C_{7}^{\prime}\right)$ in $B^{0} \rightarrow K^{* 0} \gamma$

- 'BO->K*0 deltaC7 a_- $\operatorname{Re}{ }^{\prime}-\operatorname{Re}\left(\Delta C_{7}\right)$ in $B^{0} \rightarrow K^{* 0} \gamma$

- analogously for ' $\mathrm{B}+->\mathrm{K} *+$ ' and 'Bs->phi'

- analogously for the imaginary parts of $\Delta C_{7}^{(\prime)}$ using 'Re' $\rightarrow$ ' Im'.

A Jupyter notebook to reproduce the plots in figure 3 can be found in a public repository: [71]. 
Open Access. This article is distributed under the terms of the Creative Commons Attribution License (CC-BY 4.0), which permits any use, distribution and reproduction in any medium, provided the original author(s) and source are credited.

\section{References}

[1] D. Atwood, M. Gronau and A. Soni, Mixing induced CP asymmetries in radiative B decays in and beyond the standard model, Phys. Rev. Lett. 79 (1997) 185 [hep-ph/9704272] [INSPIRE].

[2] P. Ball and R. Zwicky, Time-dependent CP Asymmetry in $B \rightarrow K^{*} \gamma$ as a (Quasi) Null Test of the Standard Model, Phys. Lett. B 642 (2006) 478 [hep-ph/0609037] [InSPIRE].

[3] F. Krüger and J. Matias, Probing new physics via the transverse amplitudes of $B^{0} \rightarrow K^{* 0}\left(\rightarrow K^{-} \pi^{+}\right) l^{+} l^{-}$at large recoil, Phys. Rev. D 71 (2005) 094009 [hep-ph/0502060] [INSPIRE].

[4] LHCb collaboration, First experimental study of photon polarization in radiative $B_{s}^{0}$ decays, Phys. Rev. Lett. 118 (2017) 021801 [arXiv: 1609.02032] [INSPIRE].

[5] F. Muheim, Y. Xie and R. Zwicky, Exploiting the width difference in $B_{s} \rightarrow \phi \gamma$, Phys. Lett. B 664 (2008) 174 [arXiv:0802.0876] [INSPIRE].

[6] F. Beaujean, C. Bobeth and D. van Dyk, Comprehensive Bayesian analysis of rare (semi)leptonic and radiative B decays, Eur. Phys. J. C 74 (2014) 2897 [Erratum ibid. C $\mathbf{7 4}$ (2014) 3179] [arXiv:1310.2478] [INSPIRE].

[7] W. Altmannshofer and D.M. Straub, New physics in $b \rightarrow s$ transitions after LHC run 1, Eur. Phys. J. C 75 (2015) 382 [arXiv:1411.3161] [INSPIRE].

[8] S. Descotes-Genon, L. Hofer, J. Matias and J. Virto, Global analysis of $b \rightarrow$ sll anomalies, JHEP 06 (2016) 092 [arXiv: 1510.04239] [INSPIRE].

[9] T. Hurth, F. Mahmoudi and S. Neshatpour, On the anomalies in the latest LHCb data, Nucl. Phys. B 909 (2016) 737 [arXiv:1603.00865] [INSPIRE].

[10] S. Descotes-Genon, D. Ghosh, J. Matias and M. Ramon, Exploring New Physics in the $C_{7}-C_{7}^{\prime}$ plane, JHEP 06 (2011) 099 [arXiv:1104.3342] [inSPIRE].

[11] D. Becirevic, E. Kou, A. Le Yaouanc and A. Tayduganov, Future prospects for the determination of the Wilson coefficient $C_{7 \gamma}^{\prime}$, JHEP 08 (2012) 090 [arXiv:1206.1502] [INSPIRE].

[12] T. Blake, G. Lanfranchi and D.M. Straub, Rare B Decays as Tests of the Standard Model, Prog. Part. Nucl. Phys. 92 (2017) 50 [arXiv:1606.00916] [inSPIRE].

[13] K.G. Chetyrkin, M. Misiak and M. Münz, Weak radiative B meson decay beyond leading logarithms, Phys. Lett. B 400 (1997) 206 [Erratum ibid. B 425 (1998) 414] [hep-ph/9612313] [INSPIRE].

[14] A.J. Buras, Climbing NLO and NNLO Summits of Weak Decays, arXiv:1102.5650 [INSPIRE].

[15] M. Benzke, S.J. Lee, M. Neubert and G. Paz, Factorization at Subleading Power and Irreducible Uncertainties in $\bar{B} \rightarrow X_{s} \gamma$ Decay, JHEP 08 (2010) 099 [arXiv:1003.5012] [INSPIRE].

[16] M. Misiak et al., Updated NNLO QCD predictions for the weak radiative B-meson decays, Phys. Rev. Lett. 114 (2015) 221801 [arXiv:1503.01789] [INSPIRE]. 
[17] M. Benzke, S.J. Lee, M. Neubert and G. Paz, Long-Distance Dominance of the CP Asymmetry in $B \rightarrow X_{s, d}+\gamma$ Decays, Phys. Rev. Lett. 106 (2011) 141801 [arXiv:1012.3167] [INSPIRE].

[18] T. Hurth, E. Lunghi and W. Porod, Untagged $\bar{B} \rightarrow X_{s+d} \gamma$ CP asymmetry as a probe for new physics, Nucl. Phys. B 704 (2005) 56 [hep-ph/0312260] [InSPIRE].

[19] Heavy Flavor Averaging Group (HFAG) collaboration, Y. Amhis et al., Averages of b-hadron, c-hadron and $\tau$-lepton properties as of summer 2014, arXiv:1412.7515 [INSPIRE].

[20] A. Bharucha, D.M. Straub and R. Zwicky, $B \rightarrow V \ell^{+} \ell^{-}$in the Standard Model from light-cone sum rules, JHEP 08 (2016) 098 [arXiv: 1503.05534] [INSPIRE].

[21] P. Ball and R. Zwicky, $B_{d, s} \rightarrow \rho, \omega, K^{*}, \phi$ decay form-factors from light-cone sum rules revisited, Phys. Rev. D 71 (2005) 014029 [hep-ph/0412079] [INSPIRE].

[22] R.R. Horgan, Z. Liu, S. Meinel and M. Wingate, Rare B decays using lattice QCD form factors, PoS (LATTICE2014) 372 [arXiv: 1501.00367] [INSPIRE].

[23] H.H. Asatryan, H.M. Asatrian, C. Greub and M. Walker, Calculation of two loop virtual corrections to $b \rightarrow s \ell^{+} \ell^{-}$in the standard model, Phys. Rev. D 65 (2002) 074004 [hep-ph/0109140] [INSPIRE].

[24] M. Beneke, T. Feldmann and D. Seidel, Systematic approach to exclusive $B \rightarrow V \ell^{+} \ell^{-}, V \gamma$ decays, Nucl. Phys. B 612 (2001) 25 [hep-ph/0106067] [INSPIRE].

[25] A.L. Kagan and M. Neubert, Isospin breaking in $B \rightarrow K^{*} \gamma$ decays, Phys. Lett. B 539 (2002) 227 [hep-ph/0110078] [INSPIRE].

[26] T. Feldmann and J. Matias, Forward backward and isospin asymmetry for $B \rightarrow K^{*} l^{+} l^{-}$ decay in the standard model and in supersymmetry, JHEP 01 (2003) 074 [hep-ph/0212158] [INSPIRE].

[27] M. Beneke, T. Feldmann and D. Seidel, Exclusive radiative and electroweak $b \rightarrow d$ and $b \rightarrow s$ penguin decays at NLO, Eur. Phys. J. C 41 (2005) 173 [hep-ph/0412400] [INSPIRE].

[28] P. Gambino and M. Misiak, Quark mass effects in $\bar{B} \rightarrow X_{s} \gamma$, Nucl. Phys. B 611 (2001) 338 [hep-ph/0104034] [INSPIRE].

[29] H.M. Asatrian, C. Greub, A. Hovhannisyan, T. Hurth and V. Poghosyan, Reduction of charm quark mass scheme dependence in $\bar{B} \rightarrow X_{s} \gamma$ at the NNLL level, Phys. Lett. B 619 (2005) 322 [hep-ph/0505068] [INSPIRE].

[30] A. Ali, B.D. Pecjak and C. Greub, B $\rightarrow V \gamma$ Decays at NNLO in SCET, Eur. Phys. J. C 55 (2008) 577 [arXiv: 0709.4422] [inSPIRE].

[31] P. Ball, G.W. Jones and R. Zwicky, $B \rightarrow V \gamma$ beyond QCD factorisation, Phys. Rev. D 75 (2007) 054004 [hep-ph/0612081] [INSPIRE].

[32] M. Dimou, J. Lyon and R. Zwicky, Exclusive Chromomagnetism in heavy-to-light FCNCs, Phys. Rev. D 87 (2013) 074008 [arXiv:1212.2242] [InSPIRE].

[33] J. Lyon and R. Zwicky, Isospin asymmetries in $B \rightarrow\left(K^{*}, \rho\right) \gamma / l^{+} l^{-}$and $B \rightarrow K l^{+} l^{-}$in and beyond the standard model, Phys. Rev. D 88 (2013) 094004 [arXiv:1305.4797] [INSPIRE].

[34] A. Khodjamirian, T. Mannel, A.A. Pivovarov and Y.M. Wang, Charm-loop effect in $B \rightarrow K^{(*)} \ell^{+} \ell^{-}$and $B \rightarrow K^{*} \gamma$, JHEP 09 (2010) 089 [arXiv:1006.4945] [INSPIRE].

[35] B. Grinstein, Y. Grossman, Z. Ligeti and D. Pirjol, The photon polarization in $B \rightarrow X \gamma$ in the standard model, Phys. Rev. D 71 (2005) 011504 [hep-ph/0412019] [INSPIRE]. 
[36] B. Grinstein and D. Pirjol, The CP asymmetry in $B^{0}(t) \rightarrow K_{S} \pi^{0} \gamma$ in the standard model, Phys. Rev. D 73 (2006) 014013 [hep-ph/0510104] [InSPIRE].

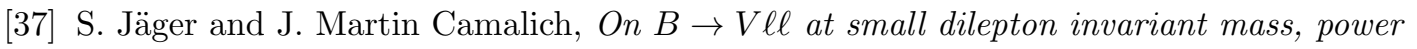
corrections and new physics, JHEP 05 (2013) 043 [arXiv: 1212.2263] [INSPIRE].

[38] S. Jäger and J. Martin Camalich, Reassessing the discovery potential of the $B \rightarrow K^{*} \ell^{+} \ell^{-}$ decays in the large-recoil region: SM challenges and BSM opportunities, Phys. Rev. D 93 (2016) 014028 [arXiv: 1412.3183] [INSPIRE].

[39] C. Bobeth, G. Hiller and G. Piranishvili, CP Asymmetries in bar $B \rightarrow \bar{K}^{*}(\rightarrow \bar{K} \pi) \bar{\ell} \ell$ and Untagged $\bar{B}_{s}, B_{s} \rightarrow \phi\left(\rightarrow K^{+} K^{-}\right) \bar{\ell} \ell$ Decays at NLO, JHEP 07 (2008) 106 [arXiv: 0805.2525] [INSPIRE].

[40] W. Altmannshofer, P. Ball, A. Bharucha, A.J. Buras, D.M. Straub and M. Wick, Symmetries and Asymmetries of $B \rightarrow K^{*} \mu^{+} \mu^{-}$Decays in the Standard Model and Beyond, JHEP 01 (2009) 019 [arXiv:0811.1214] [INSPIRE].

[41] J. Matias, F. Mescia, M. Ramon and J. Virto, Complete Anatomy of $\bar{B}_{d} \rightarrow \bar{K}^{* 0}(\rightarrow K \pi) l^{+} l^{-}$ and its angular distribution, JHEP 04 (2012) 104 [arXiv:1202.4266] [INSPIRE].

[42] LHCb collaboration, Observation of Photon Polarization in the $b \rightarrow s \gamma$ Transition, Phys. Rev. Lett. 112 (2014) 161801 [arXiv:1402.6852] [INSPIRE].

[43] M. Gronau, Y. Grossman, D. Pirjol and A. Ryd, Measuring the photon polarization in $B \rightarrow K \pi \pi \gamma$, Phys. Rev. Lett. 88 (2002) 051802 [hep-ph/0107254] [InSPIRE].

[44] E. Kou, A. Le Yaouanc and A. Tayduganov, Determining the photon polarization of the $b \rightarrow s \gamma$ using the $B \rightarrow K_{1}(1270) \gamma \rightarrow(K \pi \pi) \gamma$ decay, Phys. Rev. D 83 (2011) 094007 [arXiv: 1011.6593] [INSPIRE].

[45] E. Kou, A. Le Yaouanc and A. Tayduganov, Angular analysis of $B \rightarrow J / \psi K_{1}$ : towards a model independent determination of the photon polarization with $B \rightarrow K_{1} \gamma$, Phys. Lett. B 763 (2016) 66 [arXiv:1604.07708] [INSPIRE].

[46] M. Misiak and M. Steinhauser, Weak Radiative Decays of the B Meson and Bounds on $M_{H^{ \pm}}$ in the Two-Higgs-Doublet Model, arXiv:1702.04571 [INSPIRE].

[47] LHCb collaboration, Measurement of the ratio of branching fractions $B R\left(B_{0} \rightarrow K^{\star 0} \gamma\right) / B R\left(B_{s 0} \rightarrow \phi \gamma\right)$ and the direct $C P$ asymmetry in $B_{0} \rightarrow K^{\star 0} \gamma$, Nucl. Phys. B 867 (2013) 1 [arXiv:1209.0313] [InSPIRE].

[48] Belle collaboration, D. Dutta et al., Search for $B_{s}^{0} \rightarrow \gamma \gamma$ and a measurement of the branching fraction for $B_{s}^{0} \rightarrow \phi \gamma$, Phys. Rev. D 91 (2015) 011101 [arXiv:1411.7771] [INSPIRE].

[49] LHCb collaboration, Angular analysis of the $B^{0} \rightarrow K^{* 0} e^{+} e^{-}$decay in the low- $q^{2}$ region, JHEP 04 (2015) 064 [arXiv: 1501.03038] [INSPIRE].

[50] David Straub, flavio v0.20, (2017) https://doi.org/10.5281/zenodo.375591.

[51] HEPfit: a tool to combine indirect and direct constraints on High Energy Physics, http://hepfit.roma1.infn.it/.

[52] CLEO collaboration, S. Chen et al., Branching fraction and photon energy spectrum for $b \rightarrow s \gamma$, Phys. Rev. Lett. 87 (2001) 251807 [hep-ex/0108032] [INSPIRE].

[53] BELLE collaboration, K. Abe et al., A measurement of the branching fraction for the inclusive $B \rightarrow X_{(s)} \gamma$ decays with BELLE, Phys. Lett. B 511 (2001) 151 [hep-ex/0103042] [InSPIRE]. 
[54] Belle collaboration, A. Limosani et al., Measurement of Inclusive Radiative B-meson Decays with a Photon Energy Threshold of 1.7-GeV, Phys. Rev. Lett. 103 (2009) 241801 [arXiv: 0907.1384] [INSPIRE].

[55] BABAR collaboration, B. Aubert et al., Measurement of the $B \rightarrow X_{s} \gamma$ branching fraction and photon energy spectrum using the recoil method, Phys. Rev. D 77 (2008) 051103 [arXiv:0711.4889] [INSPIRE].

[56] BaBAR collaboration, J.P. Lees et al., Precision Measurement of the $B \rightarrow X_{s} \gamma$ Photon Energy Spectrum, Branching Fraction and Direct CP Asymmetry $A_{C P}\left(B \rightarrow X_{s+d} \gamma\right)$, Phys. Rev. Lett. 109 (2012) 191801 [arXiv:1207.2690] [InSPIRE].

[57] BABAR collaboration, J.P. Lees et al., Measurement of $B\left(B \rightarrow X_{s} \gamma\right)$, the $B \rightarrow X_{s} \gamma$ photon energy spectrum and the direct $C P$ asymmetry in $B \rightarrow X_{s+d} \gamma$ decays, Phys. Rev. D 86 (2012) 112008 [arXiv:1207.5772] [INSPIRE].

[58] BABAR collaboration, J.P. Lees et al., Exclusive Measurements of $b \rightarrow s \gamma$ Transition Rate and Photon Energy Spectrum, Phys. Rev. D 86 (2012) 052012 [arXiv:1207.2520] [InSPIRE].

[59] CLEO collaboration, T.E. Coan et al., Study of exclusive radiative B meson decays, Phys. Rev. Lett. 84 (2000) 5283 [hep-ex/9912057] [INSPIRE].

[60] Belle collaboration, M. Nakao et al., Measurement of the $B \rightarrow K^{*} \gamma$ branching fractions and asymmetries, Phys. Rev. D 69 (2004) 112001 [hep-ex/0402042] [InSPIRE].

[61] BABAR collaboration, B. Aubert et al., Measurement of Branching Fractions and CP and Isospin Asymmetries in $B \rightarrow K^{*}(892) \gamma$ Decays, Phys. Rev. Lett. 103 (2009) 211802 [arXiv: 0906.2177] [INSPIRE].

[62] M. Jung, Branching ratio measurements and isospin violation in B-meson decays, Phys. Lett. B 753 (2016) 187 [arXiv: 1510.03423] [INSPIRE].

[63] Belle collaboration, J. Wicht et al., Observation of $B_{s}^{0} \rightarrow \phi \gamma$ and Search for $B_{s}^{0} \rightarrow \gamma \gamma$ Decays at Belle, Phys. Rev. Lett. 100 (2008) 121801 [arXiv:0712.2659] [InSPIRE].

[64] D. Foreman-Mackey, D.W. Hogg, D. Lang and J. Goodman, emcee: The MCMC Hammer, Publ. Astron. Soc. Pac. 125 (2013) 306 [arXiv:1202.3665] [InSPIRE].

[65] BaBAr collaboration, B. Aubert et al., Measurement of Time-Dependent CP Asymmetry in $B^{0} \rightarrow K_{S}^{0} \pi^{0} \gamma$ Decays, Phys. Rev. D 78 (2008) 071102 [arXiv:0807.3103] [InSPIRE].

[66] Belle collaboration, Y. Ushiroda et al., Time-Dependent CP Asymmetries in $B^{0} \rightarrow K_{S}^{0} \pi^{0} \gamma$ transitions, Phys. Rev. D 74 (2006) 111104 [hep-ex/0608017] [INSPIRE].

[67] T. Aushev et al., Physics at Super B Factory, arXiv:1002.5012 [INSPIRE].

[68] T. Mannel and S. Recksiegel, Flavor changing neutral current decays of heavy baryons: The case $\Lambda_{b} \rightarrow \Lambda \gamma$, J. Phys. G 24 (1998) 979 [hep-ph/9701399] [INSPIRE].

[69] G. Hiller and A. Kagan, Probing for new physics in polarized $\Lambda_{b}$ decays at the $Z$, Phys. Rev. D 65 (2002) 074038 [hep-ph/0108074] [inSPIRE].

[70] A. Agadjanov, V. Bernard, U.-G. Meißner and A. Rusetsky, The $B \rightarrow K^{*}$ form factors on the lattice, Nucl. Phys. B 910 (2016) 387 [arXiv:1605.03386] [INSPIRE].

[71] D. Straub and A. Paul. https://github.com/DavidMStraub/paper-bvgamma-ps, DOI:10.5281/zenodo.375593. 